Western University

Scholarship@Western

Physical Therapy Publications

Physical Therapy School

$12-7-2016$

\title{
Profiles of Fatigue Severity and Variability Among Adolescents and Young Adults with Cerebral Palsy
}

Laura K. Brunton

Western University, 1brunto3@uwo.ca

Doreen J. Bartlett

Western University, djbartle@uwo.ca

Follow this and additional works at: https://ir.lib.uwo.ca/ptpub

Part of the Physical Therapy Commons

Citation of this paper:

Brunton, L. K., \& Bartlett, D. J. (2017). Profiles of fatigue severity and variability among adolescents and young adults with cerebral palsy. Fatigue: Biomedicine, Health \& Behavior, 5(1), 5-14. 


\section{Profiles of fatigue severity and variability among adolescents and young adults with cerebral palsy}

Shortened Title: Fatigue Severity and Variability in Cerebral Palsy

Laura K. Brunton ${ }^{1}$, PT, PhD, Postdoctoral Fellow, Faculty of Kinesiology, University of Calgary, Calgary AB, Canada

Doreen J. Bartlett, PT, PhD, Professor, The School of Physical Therapy, Western University, London ON, Canada

${ }^{1}$ Corresponding Author. Email: lbrunto3@uwo.ca 


\section{Profiles of fatigue severity and variability among adolescents and young adults with cerebral palsy}

\section{Abstract}

Background: Individuals with cerebral palsy (CP) experience progressive changes in functional status with the development of secondary impairments such as fatigue. Detailed accounts of the fatigue experience in CP are lacking. Purpose: This study describes the severity and variability of fatigue in CP using the Fatigue Impact and Severity Self-Assessment.

Methods: This was a descriptive cross-sectional study surveying 130 (61 males) individuals (mean age 18 years, 11 months; SD four years, six months) with CP. Analyses comprised comparisons between two groups (Gross Motor Function Classification System (GMFCS) I and II-V), frequency counts and proportions. Results: Significant differences exist between GMFCS groups for all fatigue severity questions. In addition, 38\% of individuals classified as GMFCS level I reported their average fatigue as moderate to severe and $53 \%$ experienced fatigue on three or more days in the previous week. Sixty-eight percent of individuals classified as GMFCS II-V reported their average fatigue to be moderate to severe and 78\% were fatigued on three or more days in the previous week.

Conclusions: Fatigue is a highly individualized phenomenon with significant burden for individuals with CP regardless of functional ability. Individuals in GMFCS Levels II-V reported increased fatigue severity and would benefit from specific management interventions.

Keywords: Fatigue, severity, variability, cerebral palsy, adolescents, young adults 


\section{Introduction}

Cerebral palsy (CP) is a permanent disorder of movement and posture that leads to activity limitations as a result of non-progressive disturbances that occurred in the developing fetal or infant brain.[1] Although the pathophysiology is non-progressive in nature, it is well established that individuals with CP experience progressive changes in functional status over time.[2-6] With growth and maturation children and youth with CP may develop secondary impairments. Fatigue is a common secondary impairment associated with CP [7-8] and may be connected to observed declines in physical function, including the cessation of walking.[9] Youth and adults with CP are known to be more fatigued than the general population.[10-13] Taken together, between 18-39\% of adults with CP (aged 16-80 years) are estimated to experience fatigue, and of those who are fatigued, anywhere from $12-41 \%$ are considered to be severely fatigued, as determined by a Fatigue Severity Score (FSS) $\geq 5.1 .[11-13]$

Fatigue may be related to the level of the motor impairment experienced; however, fatigue has not consistently been shown to differ by level of functional ability as measured by Gross Motor Function Classification System (GMFCS) [14] level, [12-13,15] or the Gillette Functional Assessment Questionnaire.[8] However, individuals with bilateral distribution of CP (a potential proxy measure of increased functional limitation) have been found to be more fatigued than those with unilateral involvement.[13] What is clear is that fatigue represents a significant issue for individuals with CP regardless of age and functional ability level, but 
detailed descriptions regarding self-reported severity and information about the variability of fatigue are not yet available.

\section{Current Measurement Challenges}

The lack of detailed information regarding self-reported severity of fatigue may stem from inadequate tools used to measure fatigue in this population. Many of the studies conducted to date have used measures of fatigue that are not validated for use within the CP population and/or provide only limited information about fatigue severity. For example, the FSS, although widely used to assess fatigue in samples with CP, has never been validated in this population. The FSS has been validated for use in other neurological conditions and generally demonstrates satisfactory convergent validity with varying strength of significant correlations with other measures of fatigue and health status. However, the original nine-item FSS did not withstand tests of unidimensionality, illustrating that calculating a summary score of the nine items may be inappropriate in neurological conditions.[16-17] In addition, the validity of the seven-point scoring system of the original FSS has been questioned.[16] The rating scale of the FSS ranges from one (completely disagree) to seven (completely agree) for all nine items. A score $\geq 5.1$ has been shown to be equal to two standard deviations above the mean of reference sample and has been used to classify individuals as "severely fatigued" [18] despite the fact that neither the scoring system nor any of the individual items include descriptors specifically related to severity. As a result, using the FSS provides limited information about the perceived severity and no information about the variability of fatigue experienced by individuals. 


\section{The Fatigue Impact and Severity Self-Assessment}

The unpredictable and highly variable nature of fatigue were highlighted as important by youth and young adults with $\mathrm{CP}$ in a qualitative study regarding the bodily experience of CP [19]; these issues need to be quantified and investigated further. To address some of the inadequacies of available tools to measure fatigue, the Fatigue Impact and Severity Self-Assessment (FISSA) was created and validated for youth and young adults with CP.[15] Specifically, the FISSA was created to assist with identifying individuals who are experiencing fatigue related to $\mathrm{CP}$ and to promote discussions between clinicians and their clients about fatigue and possible management strategies.[15]

The FISSA is a 37-item self-report questionnaire. The first 31 questions are generally scored using a five-point Likert scale from one (completely disagree) to five (completely agree). Questions related to the severity and variability of fatigue have a variety of scaling options and include questions related to fatigue experienced on weekdays vs. weekend days, average and cumulative fatigue over the week, and days where the most and least fatigue was experienced. Questions related to severity ask for a rating from one (no fatigue) to five (severe fatigue).[15] An impact score can be calculated by summing items one to 17 contained in the Impact Subscale (includes the severity profile) and a management score can be calculated by summing items 18-31 in the Management and Activity Modification Subscale.[15] Together the scores on both subscales produce a total score from responses to the first 31 questions and describes the fatigue experienced in terms of impact, severity and individual management. The remaining six questions are 
comprised of asking individuals about diurnal fatigue as well as open-ended questions about managing fatigue for intervention planning.[15] Although these questions are not scored as part of either subscale or the total score, they provide a starting point for the clinical conversation about fatigue management. The FISSA was validated for use with adolescents and young adults with CP. An exploratory factor analysis and a known groups validation approach provided evidence of content and construct validity of the FISSA.[15] The FISSA also demonstrated adequate test-retest reliability for adolescents and young adults with CP.[15] Use of the FISSA will provide a more detailed understanding of fatigue for individuals with $\mathrm{CP}$ as they experience it.

The purpose of this quantitative study was to describe, in detail, the severity and variability of fatigue as reported by youth and young adults with CP. The primary objective was to describe and compare responses to the severity and variability questions of the FISSA between two functionally defined groups (individuals classified as GMFCS level I and individuals classified as GMFCS levels IIV). A secondary objective of this study was to describe and compare the severity and variability responses of the FISSA between two groups defined by distribution of CP (unilateral and bilateral).

\section{Method}

This was a descriptive cross-sectional study of fatigue experienced by adolescents and young adults with CP. A total of 367 youth and young adults with CP were contacted by regular post or electronic mail as part a larger study. Potential 
participants were recruited from participating children's rehabilitation centres in Ontario, Canada, past research studies conducted by the research team, existing facebook groups for individuals with CP and through the Ontario Federation for Cerebral Palsy website and newsletter. Eligibility criteria included individuals aged 14 to 31 years of age, those who were English speaking and had the ability to complete self-report questionnaires with some degree of independence. Returned surveys completed entirely by parental proxy were excluded from the study. Diagnosis of CP was self-reported by all individuals. The survey mailing contained the FISSA, a self-report version of the GMFCS-Extended and Revised Version [20] to describe the functional abilities of respondents (self-report version available from https://www.canchild.ca) and a simple demographic questionnaire used to obtain self-reported distribution of involvement, age, sex and information regarding the amount of assistance required to complete the survey. The study followed a modified Dillman method.[21] This study received approval from the ethics review boards at Western University, McMaster University and specific clinical sites as appropriate. For the purposes of this study returning a completed questionnaire was considered implied consent.

Of the 367 individuals contacted, 163 questionnaires were returned over the course of the study, for a response rate of $44 \%$. Ten questionnaires were returned blank, eight individuals reported that they did not meet eligibility criteria and therefore did not complete the questionnaire, and a further 15 questionnaires were completed entirely by parental proxy and were excluded from the analyses. A final convenience sample of 130 youth and young adults with CP participated in the study 
by returning a questionnaire completed at least semi-independently (ranging from independent completion to having some assistance completing the questionnaire). Participant demographics are presented in Table 1 [table 1 near here].

As a result of the small number of participants in each GMFCS level, GMFCS levels were grouped together to increase the subgroup sample size and consisted of individuals who self-classified as level I (individuals who walk without limitations) separately, levels II (individuals who walk with limitations) and III (individuals who walk using a hand-held mobility device) were grouped together and levels IV (individuals who may use powered mobility due to limitations in self-mobilization) and $\mathrm{V}$ (individuals without the ability to self-mobilize) formed the third group.[20] These groups were based on an a priori hypothesis that individuals who had greater functional ability would experience less fatigue. A Kruskal-Wallis statistic was used to determine if the FISSA scores (total score, impact subscale score and management subscale score) differed by GMFCS grouping. To determine if the FISSA scores were different for individuals with unilateral versus bilateral involvement a Mann-Whitney U test was performed for the total score, impact score and management score. To determine if the responses to the severity and variability questions differed by GMFCS grouping, a Pearson's Chi-Square analysis was performed. Descriptive analysis of the survey responses comprised frequency counts and proportions by GMFCS grouping. Individuals with missing GMFCS data (6 participants) or distribution of involvement data (4 participants) were excluded from grouped analyses. Similar analyses were conducted for an additional two groups, individuals with unilateral involvement regardless of functional ability level 
and individuals with bilateral involvement regardless of functional ability level because fatigue is thought to be related to distribution of involvement. ${ }^{13}$ These results are available in a supplementary file.

\section{Results}

Individuals classified as GMFCS level I experienced significantly less fatigue than individuals classified in any other GMFCS level (II-V) $(p<0.001)$. There were no significant differences in any of the FISSA scores (total score, impact subscale or management subscale score) for individuals classified as GMFCS levels II- V. Therefore, the remaining results will be presented for two functional groups, individuals classified as GMFCS level I separately and individuals classified as GMFCS II-V as a whole. A summary of the FISSA total and subscale scores by group can be found in Table 2 [table 2 near here]. Total fatigue $\left(\chi^{2}=28.504, p<.0001\right)$, impact score $\left(\chi^{2}=34.677, \mathrm{p}<.0001\right)$ and management score $\left(\chi^{2}=13.805, \mathrm{p}<.0001\right)$ were significantly different between GMFCS groups. Total fatigue score and the impact score significantly differed between distribution of involvement groups. Specifically, individuals with bilateral distribution of CP experienced higher total fatigue $(U=1217, p=0.021)$ and higher impact of fatigue $(U=987.5, p=0.005)$ than those with unilateral distribution of CP. The management score was not significantly different between the two distribution groups $(U=1110.5, p=0.067)$. Further details of the distribution of involvement data can be found in a supplementary file (Appendix A) as the focus of this manuscript is the comparison of fatigue based on functional ability level. 


\section{Comparisons between functional groups}

Significant differences were found to exist between GMFCS grouping and all questions related to severity of fatigue, including level of fatigue on the most $\left(\chi^{2}=\right.$ $21.428, \mathrm{p}<.001)$, least $\left(\chi^{2}=14.149, \mathrm{p}=.007\right)$, and average $\left(\chi^{2}=24.025, \mathrm{p}<.0001\right)$ day. Similar results were found for the questions regarding how much of the day they were fatigued $\left(\chi^{2}=13.291, p=.01\right)$ and how many days in the previous week they were fatigued $\left(\chi^{2}=20.019, p=.006\right)$. However, questions regarding the variability of fatigue were not significantly different by GMFCS grouping, including changes in fatigue depending on time of day $\left(\chi^{2}=3.877, p=.05\right)$ and changes in fatigue depending on day of the week $\left(\chi^{2}=0.580, p=.45\right)$.

\section{GMFCS Level I}

When asked to report on the severity of their fatigue on the day in the previous week that they were most fatigued, 58\% reported having experienced moderate to severe fatigue. When asked about the day in the previous week that they were least fatigued 33\% reported some fatigue and fewer than $10 \%$ reported this to be moderate to severe fatigue. When asked to report their average level of fatigue for the previous week, 38\% reported experiencing moderate fatigue with no participant in GMFCS level I reporting their average fatigue level to be severe. Twenty-seven percent reported that on the average day they experienced no fatigue at all (Figure 1) [figure 1 near here].

Participants were asked to report, on average, how much of the day that they felt fatigued. Seventy percent responded that they were fatigued at least a quarter of the average day or more. Participants were then asked how many days in the 
previous week that they felt fatigued, the most frequently reported option was one day; however, when combined, 53\% reported experiencing fatigue on at least three or more days of the previous week. In regards to variability of fatigue, $70 \%$ reported that their fatigue changed depending on the time of day; but only 33\% reported their fatigue varied by day of the week. Of those who reported fatigue varied by time of day, $30 \%$ reported that late afternoon was when they were most fatigued, and an additional 30\% reported the evening was when their fatigue was at its worst. Notably, another $26 \%$ chose the early morning as the time when they were most fatigued. For individuals who reported that the fatigue varied by the day of the week, Monday was reported as the day where they experienced the most fatigue by $31 \%$ of the sample, followed by Tuesday and Friday, equally, with $23 \%$ of individuals reporting the most fatigue on those days (Table 3) [table 3 near here].

\section{GMFCS Levels II-V}

Regarding severity of fatigue on the day in the previous week that they were most fatigued, all reported some level of fatigue, with $86 \%$ reported having moderate to severe fatigue. When asked about their fatigue level on the day in the previous week that they were least fatigued only $38 \%$ reported no fatigue, while $33 \%$ still reported moderate to severe fatigue. When asked to report their average level of fatigue for the previous week, only one percent reported no fatigue and $68 \%$ reported experiencing moderate to severe fatigue (Figure 1).

On the average day, $92 \%$ indicated that they were fatigued at least a quarter of the day or more. Participants also reported on how many days in the previous 
week that they felt fatigued, the most frequently reported option was all seven days; however, when combined, 78\% reported experiencing fatigue on at least three or more days of the previous week. Questions about the variability of fatigue revealed that $85 \%$ felt their fatigue changed depending on the time of day, while only $41 \%$ reported their fatigue differed depending on the day of the week. Specifically, individuals felt that fatigue was at its worst in the late afternoon (48\%), followed by the evening (21.5\%), and then by the early morning (16.5\%). For those individuals who reported different levels of fatigue over the course of the week, fatigue was most commonly reported to be at its highest on Mondays (37\%) followed closely by the end of the work/school week (Friday, 34\%). Only a few individuals reported increased fatigue mid-week or on the weekend days (Table 3).

\section{Discussion}

Individuals with $\mathrm{CP}$ consistently reported substantial fatigue that may affect activities of daily living. Despite the significant role fatigue may have in functional deterioration and loss of mobility there are currently no specific interventions targeted at reducing or managing fatigue. The FISSA was created to measure fatigue in greater detail to facilitate a better understanding of the fatigue experienced by people with CP at both the individual and group level.[15] This is the first study to describe, in depth, the severity and variability of fatigue experienced by youth and young adults with CP. The results of this study demonstrate that fatigue is a highly individualized phenomenon and that fatigue is more severe and more frequently reported by individuals with increased functional disability. More than $70 \%$ of 
participants in this study reported being fatigued for at least a quarter of their average day. Similarly, more than half the participants in this study reported experiencing fatigue on three or more days in the previous week. In those with lower functional abilities (GMFCS II-V) this increased 78\% experiencing fatigue on three or more days in the previous week. Thirty-eight percent of individuals classified as GMFCS level I reported their average fatigue to be moderate to severe, highlighting that fatigue is still a significant problem for individuals with relatively mild motor impairments. Sixty-eight percent of individuals classified as GMFCS level II-V reported their average level of fatigue as moderate to severe; clearly these individuals need management strategies aimed at reducing fatigue.

\section{Future Directions}

This study provides a rich description of the fatigue experienced by individuals with $\mathrm{CP}$ and can serve as step towards intervention planning related to energy conservation strategies. A self-report measure such as the FISSA can provide a comprehensive description of fatigue to ensure all potential aspects of the condition are considered for intervention. Eken et al. [23] demonstrated that adolescents with CP have lower levels of muscular endurance than their peers. Furthermore, they demonstrated a significant relationship between muscular endurance and level of subjective fatigue,[23] this may indicate that interventions aimed at increasing muscular endurance could be beneficial for individuals reporting high levels of fatigue on the FISSA. The FISSA contains a few items that speak to the mental component of fatigue for individuals with CP; however, further information is needed about the effect of mental state on fatigue in individuals with 
$\mathrm{CP}$ and should be explored in future studies. Although fatigue has been identified as a factor contributing to loss of mobility in the early years of adulthood $[2-5,7,9]$ there is limited descriptive data regarding the severity and variability of fatigue necessary to plan interventions aimed at mitigating the consequences of fatigue.[2$3,5,9]$ A better understanding of the fatigue experienced during the years where functional decline is prevalent will help elucidate the role that fatigue may play in loss of functional ability for people with $\mathrm{CP}$ and how it can be best managed.

\section{Limitations}

This study used a convenience sampling approach that could have resulted in a selection bias. It is possible that individuals experiencing fatigue were more likely to respond to the survey and this could result in an over-estimation of the fatigue experienced by the population with $\mathrm{CP}$. This is a limitation of the current study. The treatment centres used to identify potential participants required an internal staff member to facilitate the survey mailing and the authors did not have access to any descriptors for individuals who did not respond to the survey mailing. As a result, we were unable to determine if the characteristics of responders and nonresponders were different, potentially limiting the generalizability of the findings from this study. Finally, in the validation of the FISSA, the items contained in the management scale explained only $6.2 \%$ of variance in addition to the impact scale items. Although this is a low contribution to explained variance, and thus a potential limitation, the management scale provides more in-depth clinically relevant information and was retained as part of the scale to enhance the clinical utility of the 
FISSA. Despite these limitations, the results of this study are in agreement with the available literature for both children and adults with CP.

\section{Conclusions}

A more descriptive understanding of the fatigue experienced by individuals with $\mathrm{CP}$ across the spectrum of functional abilities and the lifespan is needed to develop interventions aimed at maintaining optimal functioning for as long as possible. Decreased physical activity and increased sedentary behaviour associated with loss of mobility are risk factors for the development of chronic diseases such as obesity, cardiovascular disease and type II diabetes. Adequate fatigue management may allow individuals with CP to remain active longer, potentially delaying or preventing the onset of chronic disease and enhancing overall quality of life. This is the first study to provide detailed descriptive information about fatigue severity and the related variability for youth and young adults with CP. This information can be used to understand the fatigue experienced by an individual person when considering clinical interventions or describing groups that may benefit from the design and implementation of new interventions to reduce fatigue.

\section{Funding:}

This work was supported through a Canadian Doctoral Student Scholarship awarded to L. Brunton from the Canadian Institutes of Health Research. 
References

[1] Rosenbaum P, Paneth N, Leviton A, et al. A report: the definition and classification of cerebral palsy April 2006. Dev Med Child Neurol. 2007;49(s109):814.

[2] Andersson C, Mattsson E. Adults with cerebral palsy: a survey describing problems, needs, and resources, with special emphasis on locomotion. Dev Med Child Neurol. 2001;43:76-82.

[3] Bottos M, Feliciangeli A, Sciuto L, et al. Functional status of adults with cerebral palsy and implications for treatment of children. Dev Med Child Neurol. 2001;43:516-528.

[4] Tosi LL, Maher N, Moore DW, et al. Adults with cerebral palsy: a workshop to define the challenges of treating and preventing secondary musculoskeletal and neuromuscular complications in this rapidly growing population. Dev Med Child Neurol. 2009;51:2-11.

[5] Opheim A, Jahnsen R, Olsson E, et al. Walking function, pain, and fatigue in adults with cerebral palsy: a 7-year follow-up study. Dev Med Child Neurol. 2009;51:381388. 
[6] Hanna SE, Rosenbaum PR, Bartlett DJ, et al. Stability and decline in gross motor function among children and youth with cerebral palsy aged 2 to 21 years. Dev Med Child Neurol. 2009;51:295-302.

[7] Hilberink SR, Roebroeck ME, Nieuwstraten W, et al. Health issues in young adults with cerebral palsy: towards a life-span perspective. J Rehabil Med. 2007;39:605611.

[8] Maher C, Crettenden A, Evans K, et al. Fatigue is a major issue for children and adolescents with physical disabilities. Dev Med Child Neurol. 2015;57:742-747.

[9] Jahnsen R, Villien L, Egeland T, et al. Locomotion skills in adults with cerebral palsy. Clin Rehabil. 2004;18:309-316.

[10] Jahnsen R, Villien L, Stanghelle JK, et al. Fatigue in adults with cerebral palsy in Norway compared with the general population. Dev Med Child Neurol. 2003;45:296303.

[11] Slaman J, Bussmann J, van der Slot WM, et al. Physical strain of walking relates to activity level in adults with cerebral palsy. Arch Phys Med Rehabil. 2013;94:896901. 
[12] van der Slot WM, Nieuwenhuijsen C, van den Berg-Emons RJ, et al. Chronic pain, fatigue, and depressive symptoms in adults with spastic bilateral cerebral palsy. Dev Med Child Neurol. 2012;54:836-842.

[13] Russchen HA, Slaman J, Stam HJ, et al. Focus on fatigue amongst young adults with spastic cerebral palsy. J Neuroeng Rehabil. 2014;11:161-168.

[14] Palisano R, Rosenbaum P, Walter S, et al. Development and reliability of a system to classify gross motor function in children with cerebral palsy. Dev Med Child Neurol. 1997;39:214-223.

[15] Brunton L, Bartlett D. Construction and validation of the Fatigue Impact and Severity Self-Assessment for youth and young adults with cerebral palsy. Dev Neurorehabil. Early Online, 10.1080/17518423.2016.1189974.

[16] Burger H, Franchignoni F, Puzic N, et al. Psychometric properties of the Fatigue Severity Scale in polio survivors. Int J Rehabil Res. 2010;33:290-297.

[17] Mills R, Young C, Nicholas R, et al. Rasch analysis of the Fatigue Severity Scale in multiple sclerosis. Mult Scler J. 2009;15:81-87.

[18] Merkies I, Schmitz P, Samijn J, et al. Fatigue in immune-mediated polyneuropathies. Neurol. 1999;53:1648-1654. 
[19] Brunton LK, Bartlett DJ. The bodily experience of cerebral palsy: a journey to self-awareness. Disabil Rehabil. 2013;35:1981-1990.

[20] Palisano RJ, Rosenbaum P, Bartlett D, et al. Content validity of the expanded and revised Gross Motor Function Classification System. Dev Med Child Neurol. 2008;50:744-750.

[21] Dillman DA. Mail and internet surveys: The tailored design method. New York: Wiley; 2000.

[22] McPhee PG, Brunton LK, Timmons BW, et al. Examining fatigue in ambulatory and non-ambulatory adults with cerebral palsy: Impact and relationships with physical activity and body composition. 2016. 13p. Located at: Dev Med Child Neurol.

[23] Eken MM, Houdijk H, Doorenbosch CA, et al. Relations between muscle endurance and subjectively reported fatigue, walking capacity, and participation in mildly affected adolescents with cerebral palsy. Dev Med Child Neurol. epub ahead of print DOI: 10.1111/dmcn.13083. 
Table 1 - Participant Characteristics and Demographic Information of the Sample

\section{Characteristic}

\begin{tabular}{lll} 
& & n (\%) \\
\hline Sex $^{\mathrm{a}}$ & Male & $61(47 \%)$ \\
& Female & $68(53 \%)$
\end{tabular}

Age
Mean, years (SD)

Median

Range

I

II

III

IV

$\mathrm{V}$

Total $(n=130)$

n (\%)

$61(47 \%)$

$68(53 \%)$

$18.9(4.5)$

17

14-31

34 (27\%)

39 (32\%)

21 (17\%)

18 (14\%)

$12(10 \%)$

$6(5 \%)$

Hemiplegia

31 (25\%)

Diplegia

44 (35\%)

Triplegia

$11(9 \%)$

Quadriplegia

34 (26\%) 
GMFCS= Gross Motor Function Classification System; Note: aone participant did not report their sex; bsix participants did not report GMFCS level; cfour participants did not report distribution of involvement. 
Table 2 - Summary of Fatigue Scores by Classification Group

\begin{tabular}{|c|c|c|c|c|}
\hline & $\begin{array}{l}\text { GMFCS Ia } \\
(n=34)\end{array}$ & $\begin{array}{l}\text { GMFCS II-Va } \\
(\mathrm{n}=90)\end{array}$ & $\begin{array}{l}\text { Unilateral } \\
\mathrm{CP}^{\mathrm{b}}(\mathrm{n}=37)\end{array}$ & $\begin{array}{l}\text { Bilateral } \\
\mathrm{CP}^{\mathrm{b}}(\mathrm{n}=89)\end{array}$ \\
\hline \multicolumn{5}{|c|}{ Total Fatigue Score } \\
\hline Mean (SD) & $71(23.3)$ & $102(25.3)$ & 85 (32.1) & 99 (25.8) \\
\hline Median (Range) & $72(31-127)$ & 105 (40-149) & $91(31-147)$ & $98(37-162)$ \\
\hline \multicolumn{5}{|c|}{ Impact Scale Score } \\
\hline Mean (SD) & $32(11.4)$ & $52(15.4)$ & $41(17.8)$ & $50(16.2)$ \\
\hline Median (Range) & $31(16-62)$ & $54(20-83)$ & $37(16-78)$ & $50(17-87)$ \\
\hline \multicolumn{5}{|c|}{ Management Score } \\
\hline Mean (SD) & 37 (13.2) & $47(11.5)$ & $40(15.5)$ & $46(11.2)$ \\
\hline Median (Range) & $38(14-60)$ & $50(14-66)$ & $42(14-66)$ & $50(17-70)$ \\
\hline
\end{tabular}

GMFCS= Gross Motor Function Classification System; Note:asix participants did not report GMFCS level; bfour participants did not report distribution of involvement. 
Table 3 - Frequency of Responses to Questions Regarding Variability of Fatigue

\begin{tabular}{|c|c|c|c|}
\hline Fatigue Related Question & $\begin{array}{l}\text { Possible } \\
\text { Responses }\end{array}$ & $\begin{array}{l}\text { GMFCS I }^{\mathrm{a}} \\
(\mathrm{n}=34)\end{array}$ & $\begin{array}{l}\text { GMFCS II-V } \\
(n=90)\end{array}$ \\
\hline On average, how much of the & None & $29 \%$ & $8 \%$ \\
\hline \multirow[t]{5}{*}{ day do you feel fatigued? } & $\begin{array}{l}\text { A Quarter } \\
\text { of the Day }\end{array}$ & $47 \%$ & $42 \%$ \\
\hline & Half the Day & $18 \%$ & $31 \%$ \\
\hline & Three Quarters & $6 \%$ & $13 \%$ \\
\hline & of the Day & & \\
\hline & All Day & $0 \%$ & $6 \%$ \\
\hline For how many days last week & 1 Day & $29 \%$ & $12 \%$ \\
\hline did you feel fatigued at least & 2 Days & $18 \%$ & $10 \%$ \\
\hline \multirow[t]{5}{*}{ part of the day? } & 3 Days & $9 \%$ & $21 \%$ \\
\hline & 4 Days & $9 \%$ & $13 \%$ \\
\hline & 5 Days & $23 \%$ & $15 \%$ \\
\hline & 6 Days & $6 \%$ & $1 \%$ \\
\hline & 7 Days & $6 \%$ & $28 \%$ \\
\hline $\begin{array}{l}\text { Does your level of fatigue } \\
\text { change depending on the time }\end{array}$ & Yes & $70 \%$ & $85 \%$ \\
\hline of day? & No & $30 \%$ & $15 \%$ \\
\hline If your fatigue changes & Early Morning & $26 \%$ & $16 \%$ \\
\hline depending on time of day, what & Mid-Morning & $5 \%$ & $6 \%$ \\
\hline
\end{tabular}




\begin{tabular}{|c|c|c|c|}
\hline time of day is your fatigue the & Noon & $9 \%$ & $8 \%$ \\
\hline \multirow[t]{2}{*}{ worst? } & Late Afternoon & $30 \%$ & $48 \%$ \\
\hline & Evening & $30 \%$ & $22 \%$ \\
\hline Does your level of fatigue & Yes & $33 \%$ & $41 \%$ \\
\hline week? & No & $67 \%$ & $59 \%$ \\
\hline If your fatigue changes & Monday & $31 \%$ & $37 \%$ \\
\hline depending on day of the week, & Tuesday & $23 \%$ & $5 \%$ \\
\hline on which day are you most & Wednesday & $0 \%$ & $7 \%$ \\
\hline \multirow[t]{4}{*}{ fatigued? } & Thursday & $15 \%$ & $15 \%$ \\
\hline & Friday & $23 \%$ & $34 \%$ \\
\hline & Saturday & $8 \%$ & $2 \%$ \\
\hline & Sunday & $0 \%$ & $0 \%$ \\
\hline
\end{tabular}


Figure 1 - Fatigue Severity Profiles by Gross Motor Function Classification System (GMFCS) Level

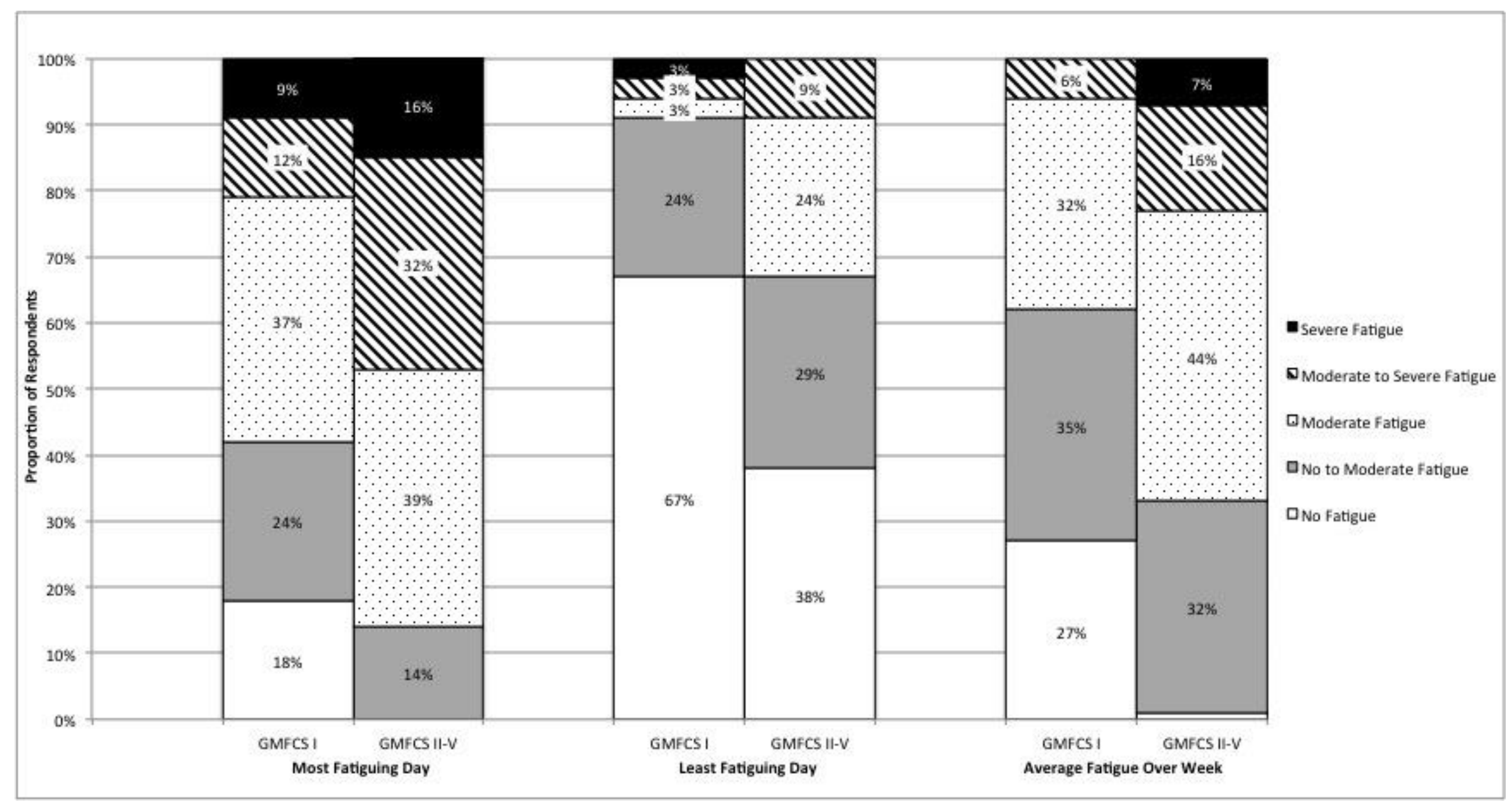

